\title{
Four-wave resonant interactions in the classical quadratic Boussinesq equations
}

\author{
M. ONORATO ${ }^{1} \dagger$, A. R. OSBORNE \\ AND D. RESIO \\ ${ }^{1}$ Dipartimento di Fisica Generale, Università di Torino, Via Pietro Giuria 1, 10125 Torino, Italy \\ ${ }^{2}$ ECMWF, Shinfield Park, Reading, UK \\ ${ }^{3}$ US Army Engineer Research \& Development Center, Vicksburg, 39180 MS, USA
}

(Received 25 January 2008 and in revised form 9 September 2008)

We investigate theoretically the irreversibile energy transfer in flat bottom shallow water waves. Starting from the oldest weakly nonlinear dispersive wave model in shallow water, i.e. the original quadratic Boussinesq equations, and by developing a statistical theory (kinetic equation) of the aforementioned equations, we show that the four-wave resonant interactions are naturally part of the shallow water wave dynamics. These interactions are responsible for a constant flux of energy in the wave spectrum, i.e. an energy cascade towards high wavenumbers, leading to a power law in the wave spectrum of the form of $k^{-3 / 4}$. The nonlinear time scale of the interaction is found to be of the order of $(h / a)^{4}$ wave periods, with $a$ the wave amplitude and $h$ the water depth. We also compare the kinetic equation arising from the Boussinesq equations with the arbitrary-depth Hasselmann equation and show that, in the limit of shallow water, the two equations coincide. It is found that in the narrow band case, both in one-dimensional propagation and in the weakly two-dimensional case, there is no irreversible energy transfer because the coupling coefficient in the kinetic equation turns out to be identically zero on the resonant manifold.

\section{Introduction}

A part of the wave forecasting community shares the common thinking that, while the four-wave (resonant) interactions are relevant in deep water, the three-wave interactions become the main nonlinear mechanism of energy transfer as the waves travel into shallow areas. Therefore, it appears that, when waves travel in shallow water, the four-wave kinetic equation (the Hasselmann equation based on the fourwave resonant interactions) is not the appropriate tool for investigation and usually different approaches are preferred (see Cavaleri et al. 2007, for a recent review, and references therein): (i) the computation of the quasi-resonant triads (see, for example Eldeberky 1996), (ii) the deterministic modelling (Boussinesq equations or higher order, see Madsen and Schaffer 1998) and (iii) the evolution equations for spectra and bispectra (see, for example Herbers and Burton 1997). Each of these methods furnishes reasonable results when compared to experimental data in shallow water but none of them can be used as a tool for making statistical predictions in infinite water depth. As a matter of fact, it appears from most of the existing literature that,

$\dagger$ Email address for correspondence: onorato@ph.unito.it 
as waves propagate from deep to shallow water, different models have to be used for an appropriate forecasting.

In this paper, our aim is to show that the nonlinear transfer in shallow water (constant depth) is not so different from the deep water one: in both cases it is ruled by a four-wave kinetic equation. We will show that a theory based on fourwave resonant interaction in shallow water can be useful for understanding some issues related to the energy transfer. In order to do that we concentrate our analysis on the simplest (and probably the oldest) deterministic shallow water model, i.e. the Boussinesq equations in flat bottom conditions; we will show analytically the following results:

(i) Exact four-wave resonant interactions are included in the flat bottom quadratic Boussinesq equations;

(ii) The typical nonlinear time scale, $\tau_{4 N L}$, of the four-wave kinetic equation in shallow water is of the order of $1 /(a / h)^{4}$ wave periods;

(iii) The four-wave kinetic equation derived from the Boussinesq equations is nothing but the limit of the arbitrary depth Hasselmann equation as $k h \rightarrow 0$; in other words, the Hasselmann equation is 'compatible' with the Boussinesq equations in flat bottom conditions.

The derivation of the above results also offers us the possibility to discuss some more technical issues related to the 'integrability' of wave systems in shallow water. We show that, if the narrow-band approximation is taken, the Zakharov equation in shallow water reduces to the shallow water Davey-Stewartson equations. This system of equations is known to be integrable and, as a consequence, it does not admit a net flux of energy or wave action across the wave spectrum. The results are reported in $\S 4$.

We also concentrate our analysis on the equilibrium range in wave spectra: in deep water it is well accepted that the inertial range in the wave spectrum is characterized by a power law of the form $\omega^{-4}$. As the water becomes shallower, it is plausible that such a law could change (this is because the coupling coefficient in the multiple integral in the nonlinear source term is a function also of the water depth). It has already been shown by Zakharov (1999) that, in the limit of shallow water, the power law in the wave spectrum should be of the form $\omega^{-4 / 3}$. This result, which has been observed experimentally by Smith and Vincent (2003) and by Kaihatu et al. (2007), is perfectly consistent with a dimensional analysis of our shallow water four-wave kinetic equation derived from the deterministic Boussinesq equations. Discussion and conclusions are reported in $\S 7$.

We mention that the present manuscript has been inspired by the following references: Zakharov (1998, 1999) and Janssen and Onorato (2007), where the first ideas on the comprehension of the four-wave resonant interactions in shallow water have been reported. In the past, there have been other attempts to address such problems (Herterich and Hasselmann 1980; Lin and Perrie 1997); nevertheless these approaches have failed either because the role of the mean flow has not been properly considered or the shallow water limit has not been taken properly. As will be shown in this paper, taking the shallow water limit is not an easy task; some small denominators appear and, if not properly treated, can lead to wrong results (Janssen and Onorato 2007; Gorman 2003).

In order to avoid any confusion and clarify the role of the present paper with respect to the existing literature, we underline that our goal here is not to develop a new model or to validate the old Boussinesq equations (or the corresponding kinetic equation); we do not even intend to develop a model which is valid from deep to 
Four-wave resonant interactions in the classical quadratic Boussinesq equations 265

shallow water as in Janssen, Herbers \& Battjes (2006) or Madsen, Bingham \& Liu (2002). Our main aim is to show that even the lowest order quadratic Boussinesq equations include naturally the four-wave resonant interactions mechanism as a nonlinear energy transfer process; if desired, the same procedure can be applied to any higher order Boussinesq model (Madsen and Schaffer 1998), increasing the accuracy of the four-wave interactions as waves propagate towards deeper water.

\section{The Boussinesq equations and four-wave interactions}

The Boussinesq equations have been a major tool for studying the dynamics of shallow water waves. They have been derived from the Euler equations in the limit of shallow water and small amplitude waves. In particular two small parameters are usually introduced: the first one is $\beta=(k h)^{2}$ where $k$ corresponds to wavenumbers and $h$ is the fluid depth; the second one is $\alpha=a / h$ where $a$ is a typical wave amplitude. The starting point of our analysis is the classical quadratic Boussinesq equations as given in text books (see Whitham 1974; Mei 2000):

$$
\begin{gathered}
\eta_{t}+\nabla \cdot[(\eta+h) \overline{\boldsymbol{u}}]=0, \\
\overline{\boldsymbol{u}}_{t}+\overline{\boldsymbol{u}} \cdot \nabla \overline{\boldsymbol{u}}+g \nabla \eta-\frac{h^{2}}{3} \nabla \nabla \cdot \overline{\boldsymbol{u}}_{t}=0 .
\end{gathered}
$$

Here $\overline{\boldsymbol{u}}=\overline{\boldsymbol{u}}(x, y, t)$ is the depth integrated horizontal velocity; $\eta=\eta(x, y, t)$ is the surface elevation and $g$ is gravity. For the derivation of (2.1) and (2.2) one needs to make the hypothesis that $\beta \sim \alpha \ll 1$ and to neglect in the expansion terms of the order of $\alpha \beta, \beta^{2}$. Note that, under the hypothesis that waves propagate in only one direction, the celebrated Korteweg and De Vries equation can be derived (Whitham 1974).

Equation (2.2) can be written for the velocity potential using $\overline{\boldsymbol{u}}=\nabla \phi$ and the resulting equation reads:

$$
\phi_{t}+\frac{1}{2}(\nabla \phi)^{2}+g \eta-\frac{h^{2}}{3} \nabla^{2} \phi_{t}=0 .
$$

Equations (2.1) and (2.3) are our starting point for the analysis.

\subsection{The Boussinesq equations in Fourier space}

We now introduce the Fourier transform of $\eta$ as

$$
\eta=\int \hat{\eta}_{k} \mathrm{e}^{\mathrm{i} k \cdot x} \mathrm{~d} \boldsymbol{k}
$$

where $\boldsymbol{k}=\left(k_{x}, k_{y}\right), \boldsymbol{x}=(x, y)$ and $\hat{\eta}_{\boldsymbol{k}}=\hat{\eta}(\boldsymbol{k}, t)$. A similar definition holds for the velocity potential. A straightforward introduction of the Fourier transform in (2.1) and (2.3) leads to the following equations:

$$
\begin{aligned}
& \frac{\partial \hat{\eta}_{0}}{\partial t}-h k_{0}^{2} \hat{\phi}_{0}=\int\left(L_{0,1,2} \hat{\phi}_{1} \hat{\eta}_{2}+L_{0,2,1} \hat{\phi}_{2} \hat{\eta}_{1}\right) \delta\left(\boldsymbol{k}_{1}+\boldsymbol{k}_{2}-\boldsymbol{k}_{0}\right) \mathrm{d} \boldsymbol{k}_{12} \\
& \gamma_{0} \frac{\partial \hat{\phi}_{0}}{\partial t}+g \hat{\eta}_{0}=\int L_{1,2,0} \hat{\phi}_{1} \hat{\phi}_{2} \delta\left(\boldsymbol{k}_{1}+\boldsymbol{k}_{2}-\boldsymbol{k}_{0}\right) \mathrm{d} \boldsymbol{k}_{12},
\end{aligned}
$$

where $L_{0,1,2}=L\left(\boldsymbol{k}_{0}, \boldsymbol{k}_{1}, \boldsymbol{k}_{2}\right)=\left(\boldsymbol{k}_{0} \cdot \boldsymbol{k}_{1}\right) / 2$ and

$$
\gamma(k)=1+\frac{k^{2} h^{2}}{3}
$$

with $k=\sqrt{k_{x}^{2}+k_{y}^{2}}$ 


\subsection{Linear equations and wave action variable}

We now consider the linearized equations (2.5) and (2.6). It is straightforward to show that the resulting dispersion relation is given by

$$
\omega_{k}=c_{0} \frac{k}{\left(1+k^{2} h^{2} / 3\right)^{1 / 2}},
$$

where $c_{0}=\sqrt{g h}$. It is interesting to note that if one introduces a wave action variable $a_{k}=a(k, t)$ related to the surface elevation and velocity potential, respectively, as

$$
\begin{aligned}
& \hat{\eta}_{k}=\left(\frac{\omega_{k}}{2 g}\right)^{1 / 2}\left(a_{k}+a_{-k}^{*}\right), \\
& \hat{\phi}_{k}=-\mathrm{i} \frac{1}{\gamma_{k}}\left(\frac{g}{2 \omega_{k}}\right)^{1 / 2}\left(a_{k}-a_{-k}^{*}\right),
\end{aligned}
$$

the coupled linear equations reduce to a single equation:

$$
\frac{\partial a_{k}}{\partial t}+\mathrm{i} \omega_{k} a_{k}=0
$$

that corresponds to an infinite number of decoupled linear oscillators.

\subsection{Nonlinear equations}

We now turn our attention to the nonlinear problem and use the relations between wave action, surface elevation and velocity potential in the nonlinear case. In order to avoid higher order nonlinear dispersive terms we use $\omega_{k}=\sqrt{g h} k$ in the right-hand side of (2.9) and (2.10) to get

$$
\begin{aligned}
\left(\frac{\omega_{k}}{2 g}\right)^{1 / 2} & \simeq \frac{1}{\sqrt{2}}\left(\frac{h}{g}\right)^{1 / 4} k^{1 / 2}, \\
\frac{1}{\gamma_{k}}\left(\frac{g}{2 \omega_{k}}\right)^{1 / 2} & \simeq \frac{1}{\sqrt{2}}\left(\frac{g}{h}\right)^{1 / 4} \frac{1}{k^{1 / 2}} .
\end{aligned}
$$

Applying the same procedure as for the linear case we obtain the following equation:

$$
\begin{aligned}
\frac{\partial a_{0}}{\partial t}+\mathrm{i} \omega_{0} a_{0}= & -\mathrm{i} \int V_{0,1,2}\left[a_{1} a_{2} \delta\left(\boldsymbol{k}_{0}-\boldsymbol{k}_{1}-\boldsymbol{k}_{2}\right)+2 a_{1}^{*} a_{2} \delta\left(\boldsymbol{k}_{0}-\boldsymbol{k}_{1}+\boldsymbol{k}_{2}\right)\right. \\
& \left.+a_{1}^{*} a_{2}^{*} \delta\left(\boldsymbol{k}_{0}+\boldsymbol{k}_{1}+\boldsymbol{k}_{2}\right)\right] \mathrm{d} \boldsymbol{k}_{12},
\end{aligned}
$$

where

$$
V_{0,1,2}=\frac{1}{4 \sqrt{2}}\left(\frac{g}{h}\right)^{1 / 4} \frac{\left(\boldsymbol{k}_{1} \cdot \boldsymbol{k}_{2}\right) k_{0}+\left(\boldsymbol{k}_{0} \cdot \boldsymbol{k}_{2}\right) k_{1}+\left(\boldsymbol{k}_{0} \cdot \boldsymbol{k}_{1}\right) k_{2}}{\left(k_{0} k_{1} k_{2}\right)^{1 / 2}} .
$$

A similar evolution equation has also been found by Zakharov (1999) starting from the arbitrary depth Euler equations in the limit of shallow water.

\section{Four-wave resonant interactions in the Boussinesq equations}

\subsection{Removing three-wave non-resonant interaction with multiple scale expansion} Apart from the case of generation of long waves which is discussed in the Appendix, three-wave interactions are never exactly resonant; therefore, it is possible to remove the quadratic nonlinearity from (2.14). There are different ways to do it. If the system is Hamiltonian it is natural to use the so-called canonical transformation, i.e. a mapping 
Four-wave resonant interactions in the classical quadratic Boussinesq equations 267

to a different variable which evolves only on the time scale of four-wave interactions which may be resonant; this methodology is explained in detail in Krasitskii (1994). The other possibility consists of using the so-called multiple scale expansion; this method does not require that the system be Hamiltonian, and it has been used in Zakharov (1968) to derive originally the Zakharov equation. The resulting equations are not necessarily Hamiltonian. Note, however, that in the multiple scale method one has also some degrees of freedom which allows one to get the final coupling coefficients with the desired symmetries. Equation (2.14) is in Hamiltonian form therefore any method can be used. Here we have used the method of multiple scales because it can be used also in the cases where the primitive equations do not have a Hamiltonian structure and therefore can be for example extended to the case of variable depth. The idea behind the method of the multiple scales is to let variable $a_{k}(t)$ be a function of slower time scales, i.e. $a_{k}(t, \tau)$ with $\tau=\epsilon^{2} t$ and look for an evolution equation for the slow amplitude variable. We look for a solution of (2.14) of the form

$$
a_{k}(t, \tau)=b_{k}(t, \tau)+\epsilon b_{k}^{(1)}(t, \tau)+\epsilon^{2} b_{k}^{(2)}(t, \tau)+\cdots .
$$

Here $b_{k}$ represents the amplitude of the free modes and $b_{k}^{(1)}$ and $b_{k}^{(2)}$ correspond to the amplitude of bound modes that can be written as a function of $b$. Inserting this solution in (2.14) and recalling that $\partial / \partial t=\partial / \partial t+\epsilon^{2} \partial / \partial \tau$, we get the evolution equation for free waves in the shallow water limit

$$
\frac{\partial b_{0}}{\partial t}+\mathrm{i} \omega_{0} b_{0}=-\mathrm{i} \int T_{0123} b_{1}^{*} b_{2} b_{3} \delta\left(\boldsymbol{k}_{0}+\boldsymbol{k}_{1}-\boldsymbol{k}_{2}-\boldsymbol{k}_{3}\right) \mathrm{d} \boldsymbol{k}_{123},
$$

where $T_{1,2,3,4}$ has the following form:

$$
\begin{aligned}
T_{1,2,3,4}= & -V_{1,3,1-3} V_{4,2,4-2}\left[\frac{1}{\omega_{3}+\omega_{1-3}-\omega_{1}}+\frac{1}{\omega_{2}+\omega_{4-2}-\omega_{4}}\right] \\
& -V_{2,3,2-3} V_{4,1,4-1}\left[\frac{1}{\omega_{3}+\omega_{2-3}-\omega_{2}}+\frac{1}{\omega_{1}+\omega_{4-1}-\omega_{4}}\right] \\
& -V_{1,4,1-4} V_{3,2,3-2}\left[\frac{1}{\omega_{4}+\omega_{1-4}-\omega_{1}}+\frac{1}{\omega_{2}+\omega_{3-2}-\omega_{3}}\right] \\
& -V_{2,4,2-4} V_{3,1,3-1}\left[\frac{1}{\omega_{4}+\omega_{2-4}-\omega_{2}}+\frac{1}{\omega_{1}+\omega_{3-1}-\omega_{3}}\right] \\
& -V_{1+2,1,2} V_{3+4,3,4}\left[\frac{1}{\omega_{1+2}-\omega_{1}-\omega_{2}}+\frac{1}{\omega_{3+4}-\omega_{3}-\omega_{4}}\right] \\
& -V_{-1-2,1,2} V_{-3-4,3,4}\left[\frac{1}{\omega_{1+2}+\omega_{1}+\omega_{2}}+\frac{1}{\omega_{3+4}+\omega_{3}+\omega_{4}}\right],
\end{aligned}
$$

where $V_{1,2,3}$ is given in (2.15) The coupling coefficient is the result of six contributions. The first four will be called quasi-singular; this is because, as will be shown in the next section, in the narrow-band approximation the denominator may vanish and such a limit should therefore be taken with care. The last two terms do not have any small denominator and will be called regular terms. Equation (3.2) will be named as the shallow water Zakharov equation; it rules the deterministic evolution of free modes in flat bottom shallow water. Equation (3.2) will represent the starting point for developing the kinetic equation. 


\section{On certain limits of the shallow water Zakharov equation}

Here we will consider the narrow-band approximation of the shallow water Zakharov equation for one- and two-dimensional propagation. This will help us in understanding the properties of integrability and the effective nonlinear transfer in shallow water. It will be shown that in the narrow-band limit there is no energy transfer because the resulting equations are integrable and the coupling coefficient in the kinetic equation, $T_{1,2,3,4}^{2}$, turns out to be exactly zero when calculated on the resonant manifold.

\subsection{On the long crested narrow-band approximation: the nonlinear Schroedinger equation}

We now consider the long crested and narrow-band approximation of the Zakharov equation. In order to write explicitly the equation, the coupling coefficient should be worked out for the case of a single wave, i.e. $T_{0,0,0,0}$. A naive attempt to calculate such a coefficient, taking the narrow-band limit of each of the contributions to $T_{0,0,0,0}$ fails because (i) the denominators contain zeros (which should be properly treated) and (ii) for each of the first four (out of six) contributions the narrow-band limit depends on the sign of the difference wave vector. This was already noted in Janssen and Onorato (2007), for example if one attempts to calculate the narrow-band approximation of the first contribution, it turns out that it depends on the sign of the difference vector $\boldsymbol{d}=\boldsymbol{k}_{1}-\boldsymbol{k}_{3}=\boldsymbol{k}_{4}-\boldsymbol{k}_{2}$, which is of no help unless one specifies an ordering of the vectors. Therefore, some caution must be taken to evaluate $T_{0,0,0,0}$.

In order to properly consider the narrow-band approximation, it is necessary to combine the first and fourth contributions and the second and third (the fifth and sixth contributions do not show any apparent singularity and can be calculated directly). This is due to the fact that, if one considers these contributions together, the result is independent of the vector $\boldsymbol{d}$ but depends on $|\boldsymbol{d}|$ and the limit of $|\boldsymbol{d}|$ going to zero can be safely taken. In order to show explicitly the calculation we consider the following contribution:

$$
C_{1,2,3,4}=-V_{1,3,1-3} V_{4,2,4-2} \frac{1}{\omega_{3}+\omega_{1-3}-\omega_{1}}-V_{2,4,2-4} V_{3,1,3-1} \frac{1}{\omega_{1}+\omega_{3-1}-\omega_{3}},
$$

which, after using the definition of $V_{1,2,3}$ in (2.15) and considering waves propagating in one direction with $\boldsymbol{k}_{i}=\left(k_{0}+\varepsilon_{i}, 0\right)$ with $k_{0}$ positive and $\varepsilon_{i}$ a small number which, in the narrow-band approximation, tends to zero, (4.1) becomes

$$
C_{\varepsilon_{1}, \varepsilon_{2}, \varepsilon_{3}, \varepsilon_{4}}=-\frac{1}{16}\left(\frac{g}{h}\right)^{1 / 2} k_{0}^{2}|\boldsymbol{d}| \frac{5 \omega_{1-3}+4 \operatorname{sign}[\boldsymbol{d}]\left(\omega_{1}-\omega_{3}\right)}{\omega_{1-3}^{2}-\left(\omega_{1}-\omega_{3}\right)^{2}},
$$

where we still have explicitly $\boldsymbol{d}=\boldsymbol{k}_{1}-\boldsymbol{k}_{3}$. Even though we are in the one-dimensional case, we still have used the vector notation because the sign of $\boldsymbol{d}$ depends on the difference $\varepsilon_{1}-\varepsilon_{3}$, whose sign is not a priori known.

The dispersion relation can be used directly to calculate $\omega_{1}-\omega_{3}$ and $\omega_{1-3}$ as a function of $|\boldsymbol{d}|$

$$
\omega_{1}-\omega_{3}=\sqrt{g h}\left[\operatorname{sign}[\boldsymbol{d}]|\boldsymbol{d}|\left(1-\frac{h^{2} k_{0}^{2}}{2}\right)-\frac{h^{2}}{6}(\operatorname{sign}[\boldsymbol{d}]|\boldsymbol{d}|)^{3}\right]
$$

and

$$
\omega_{1-3}=\sqrt{g h}\left(|\boldsymbol{d}|-\frac{h^{2}}{6}|\boldsymbol{d}|^{3}\right)
$$


Four-wave resonant interactions in the classical quadratic Boussinesq equations 269

Plugging these relations into (4.2), the sign function disappears from the equation and upon taking the limit of $|\boldsymbol{d}| \rightarrow 0$, we obtain the final result

$$
C_{0,0,0,0}=-\frac{9-2 h^{2} k_{0}^{2}}{4 h^{3}\left(4-h^{2} k_{0}^{2}\right)} \simeq-\frac{9}{16 h^{3}},
$$

where higher order terms in the dispersive parameter $k_{0} h$ have been neglected. Similar calculations can be performed to the remaining three quasi-singular terms; so that the total contribution from such quasi-singular terms is $-9 /\left(4 h^{3}\right)$. Considering the fifth regular contribution, we obtain

$$
-V_{1+2,1,2} V_{3+4,3,4}\left[\frac{1}{\omega_{1+2}-\omega_{1}-\omega_{2}}+\frac{1}{\omega_{3+4}-\omega_{3}-\omega_{4}}\right]=\frac{9}{8 h^{3}} .
$$

No small denominators appear in the last regular contribution so that a straightforward calculation leads to

$$
\begin{aligned}
& -V_{-1-2,1,2} V_{-3-4,3,4}\left[\frac{1}{\omega_{1+2}+\omega_{1}+\omega_{2}}+\frac{1}{\omega_{3+4}+\omega_{3}+\omega_{4}}\right] \\
& =-\frac{k_{0}^{2}}{32 h\left(1-(5 / 12) h^{2} k_{0}^{2}\right)} \simeq-\frac{k_{0}^{2}}{32 h} .
\end{aligned}
$$

For very shallow water this last contribution is negligible with respect to the other contributions; as a result the total contribution from quasi-singular and regular terms is

$$
T_{0,0,0,0}=-\frac{9}{8 h^{3}} .
$$

Substituting this in the shallow water Zakharov equation (3.2) and Taylor expanding $\omega$ around wavenumber $k_{0}$ we get

$$
\omega_{k}=\omega_{0}+C_{g} \chi+\alpha \chi^{2}+\cdots,
$$

with $\chi=k-k_{0}$ and

$$
\omega_{0}=\sqrt{g h} k_{0}\left(1-k_{0}^{2} h^{2} / 6\right) \quad C_{g}=\sqrt{g h}\left(1-\frac{1}{2} k_{0}^{2} h^{2}\right) \quad \alpha=-\frac{1}{2} \sqrt{g h} h^{2} k_{0} .
$$

Now, defining

$$
\psi_{k}=\left(\frac{2 c_{0} k_{0}}{g}\right)^{1 / 2} b_{k} e \mathrm{e}^{\mathrm{i} \omega_{0} t}
$$

and writing the resulting Zakharov equation in physical space, we obtain the (defocusing) nonlinear Schroedinger (NLS) equation in shallow water

$$
\frac{\partial \psi}{\partial t}+C_{g} \frac{\partial \psi}{\partial x}-\mathrm{i} \alpha \frac{\partial^{2} \psi}{\partial x^{2}}-\mathrm{i} \sigma|\psi|^{2} \psi=0,
$$

with

$$
\sigma=\frac{9}{16} \frac{c_{0}}{h^{4} k_{0}}
$$

This result is identical to the one obtained by taking the shallow water limit of the arbitrary-depth NLS equation (see Hasimoto and Ono 1972; Mei 2000). The resulting NLS equation is known to be integrable, therefore it does not admit an irreversible transfer of energy. 
4.2. The weakly two-dimensional case: the Davey-Stewartson equations

We now let the wavetrain has a weak directional spreading, i.e. we allow for a weak perturbation in the $y$-direction with $\left(k_{y} / k_{x}\right)^{2} \ll 1$ which is assumed to be of the same order as the shallow water parameter $(k h)^{2}$. The procedure for calculating the coupling coefficient is similar to the one described in the previous section with the only complication that wave vectors now are written in the following way $\boldsymbol{k}_{i}=\left(k_{0}+\chi_{i}, \mu_{i}\right)$ with $\chi_{i}$ and $\mu_{i}$ small; therefore, the vector $\boldsymbol{d}$ has both $x$ and $y$ components. Carrying out the calculation for $C_{\left\{k_{0}+\chi_{i}, \mu_{i}\right\}}$ to the leading-order approximation we obtain

$$
\begin{aligned}
C_{\left\{k_{0}+\chi_{i}, \mu_{i}\right\}} & =-\frac{1}{16}\left(\frac{g}{h}\right)^{1 / 2} k_{0}^{2}\left|\boldsymbol{d}_{x}\right| \frac{5 \omega_{1-3}+4 \operatorname{sign}\left[\boldsymbol{d}_{x}\right]\left(\omega_{1}-\omega_{3}\right)_{x}}{\left(\omega_{1-3}^{2}-\left(\omega_{1}-\omega_{3}\right)^{2}\right)_{x}+c_{0}\left(\omega_{1-3}\right)_{x}\left(\left(\mu_{1}-\mu_{3}\right)^{2} /\left|\chi_{1}-\chi_{3}\right|\right)}= \\
& =-\frac{9 k_{0}^{2}}{16 h}\left[\frac{1}{\left(k_{0} h\right)^{2}+\left(\left(\mu_{1}-\mu_{3}\right)^{2} /\left(\chi_{1}-\chi_{3}\right)^{2}\right)}\right],
\end{aligned}
$$

where we have used the relations $\chi_{1}+\chi_{2}=\chi_{3}+\chi_{4}$ and $\mu_{1}+\mu_{2}=\mu_{3}+\mu_{4}$; the subscript $x$ indicates that these quantities are calculated for the one-dimensional case as in the previous section. A similar calculation can be performed on the remaining quasi-singular terms. The first of the regular terms takes the following form:

$$
-V_{1+2,1,2} V_{3+4,3,4}\left[\frac{1}{\omega_{1+2}-\omega_{1}-\omega_{2}}+\frac{1}{\omega_{3+4}-\omega_{3}-\omega_{4}}\right]=\frac{9}{8 h^{3}},
$$

while the last regular term is of higher order. The final form of the coupling coefficient becomes

$$
\begin{aligned}
T_{\left\{k_{0}+\chi_{i}, \mu_{i}\right\}}= & \frac{9 k_{0}^{2}}{8 h}\left[\frac{1}{\left(k_{0} h\right)^{2}}-\left(\frac{1}{\left(k_{0} h\right)^{2}+\left(\left(\mu_{1}-\mu_{3}\right)^{2} /\left(\chi_{1}-\chi_{3}\right)^{2}\right)}\right)\right. \\
& \left.-\left(\frac{1}{\left(k_{0} h\right)^{2}+\left(\left(\mu_{2}-\mu_{3}\right)^{2} /\left(\chi_{2}-\chi_{3}\right)^{2}\right)}\right)\right]
\end{aligned}
$$

The dispersion relation then becomes

$$
\omega_{k}=\omega_{0}+C_{g} \chi+\alpha \chi^{2}+\beta \mu^{2},
$$

with

$$
\beta=\frac{c_{0}}{2 k_{0}} \text {. }
$$

The coupling coefficient in (4.16) have an incredibly remarkable property: it turns out to be exactly zero on the resonant manifold, i.e. in that region of space that satisfies the following relations:

$$
\omega_{1}+\omega_{2}=\omega_{3}+\omega_{4}, \quad \chi_{1}+\chi_{2}=\chi_{3}+\chi_{4}, \quad \mu_{1}+\mu_{2}=\mu_{3}+\mu_{4} .
$$

In order to prove that we eliminate $\chi_{2}$ and $\mu_{2}$ from the above expressions and solve the resulting quadratic equation for $\chi_{1}$ to obtain

$$
\chi_{1}=\frac{1}{2}\left(\chi_{3}+\chi_{4}\right) \pm \frac{1}{2} \frac{\sqrt{4\left(\mu_{1}-\mu_{3}\right)\left(\mu_{1}-\mu_{4}\right)+\left(h k_{0}\right)^{2}\left(\chi_{3}-\chi_{4}\right)^{2}}}{k_{0} h} .
$$

Inserting this relation into the coupling coefficient (4.16), after some algebraic manipulation, it turns out that the coupling coefficient is exactly zero. This result is related to the fact that the shallow water Zakharov equation obtained in the weakly two-dimensional case belongs to the family of integrable equations; for this class of equations it has been shown by Zakharov and Schulman (1988) (see also 
Zakharov 1998) that the coupling coefficient calculated on the resonant manifold is exactly zero, therefore there is no net transfer of energy. Indeed, it is straightforward to show that the resulting equation corresponds to the shallow water Davey-Stewartson equations which are known to be integrable. The arbitrary depth Davey-Stewartson equations have been derived by Davey and Stewartson in 1974 using the method of the multiple scale, directly applied to the Euler equations (see also the derivation from the Zakharov equation in Stiassnie and Shemer 1984). In the shallow water limit these equations have the following form:

$$
\begin{gathered}
\frac{\partial \psi}{\partial t}+C_{g} \frac{\partial \psi}{\partial x}-\mathrm{i} \alpha \frac{\partial^{2} \psi}{\partial x^{2}}+\mathrm{i} \beta \frac{\partial^{2} \psi}{\partial y^{2}}=\mathrm{i} \mu|\psi|^{2} \psi+\mathrm{i} \nu \psi \frac{\partial \phi}{\partial x} \\
\left(k_{0} h\right)^{2} \frac{\partial^{2} \phi}{\partial x^{2}}+\frac{\partial^{2} \phi}{\partial y^{2}}=\rho \frac{\partial|\psi|^{2}}{\partial x}
\end{gathered}
$$

with

$$
\rho=-3 c_{0} /\left(2 h^{2}\right), \quad \mu=-\frac{9}{8} \frac{c_{0}}{h^{4} k_{0}}, \quad \nu=-\frac{3}{2} k_{0} .
$$

Note that in the case where there is no dependence on the $y$ coordinate, the equations reduce to the defocusing NLS equation described in the previous section in (4.12). In order to show that the shallow water weakly two-dimensional Zakharov equation corresponds to the above equation it is sufficient to re-write (4.21) in Fourier space by simply applying the Fourier Transform definition. The equation for the mean flow can be solved directly and can be substituted in the equation for the envelope. The resulting coupling coefficient corresponds exactly to the one in (4.16) provided the relation (4.11) is used.

\section{The statistical description of the shallow water Zakharov equation}

Starting from the deterministic Boussinesq equations, in $\S 2$ we have derived an evolution equation (3.2) on a slower time scale which we have called the Zakharov equation in shallow water. It is well known that such an equation is the starting point for the statistical description of water waves. In other words, from (3.2) it is possible to derive an evolution equation for the wave action spectral density. The procedure is standard and requires the following approximations:

(i) Homogeneity of the wave field: there exists a portion of the ocean where the statistical properties of the surface elevation are space independent; this region should be much larger than the correlation length of the wave field.

(ii) The random phase approximation for free waves: the free waves obeying the Zakharov equations should be uncorrelated. This hypothesis is the basis of the closure (just like in turbulence) and allows one to split higher order correlators (which naturally arise once one is interested in writing an evolution equation for the spectrum in a nonlinear wave system) as a combination of the product of lower order correlators.

The resulting evolution equation for the wave action spectral density function $<b_{0} b_{1}^{*}>=N\left(\boldsymbol{k}_{0}, t\right) \delta\left(\boldsymbol{k}_{1}-\boldsymbol{k}_{0}\right)$, where brackets denote ensemble averaging, has the following form:

$$
\begin{aligned}
\frac{\partial N_{0}}{\partial t}= & 4 \pi \int\left|T_{0,1,2,3}\right|^{2} N_{0} N_{1} N_{2} N_{3}\left(\frac{1}{N_{0}}+\frac{1}{N_{1}}-\frac{1}{N_{2}}-\frac{1}{N_{3}}\right) \\
& \delta\left(\boldsymbol{k}_{0}+\boldsymbol{k}_{1}-\boldsymbol{k}_{2}-\boldsymbol{k}_{3}\right) \delta\left(\omega_{0}+\omega_{1}-\omega_{2}-\omega_{3}\right) \mathrm{d} \boldsymbol{k}_{123} .
\end{aligned}
$$


This is the four-wave kinetic equation derived from the deterministic Boussinesq equations, it is relevant to notice that it has the same form as the Hasselmann equation in deep water except from the fact that the dispersion relation is different and the coupling coefficient in the multiple integral is also different.

In order to give an estimate of the nonlinear time scale of the four-wave resonant interactions in shallow water, we consider the fact that at the leading non-trivial order the coupling coefficient $T_{0,1,2,3}$ scales as $k^{2} / h$, therefore we have

$$
\tau_{4 N L} \sim \frac{\omega}{N^{2} T^{2} k^{4}} \sim \frac{1}{\omega}\left(\frac{1}{a / h}\right)^{4} .
$$

In very shallow water the time scale can be very fast! Note that in deep water the nonlinear time scale is of the order of $1 /\left(\omega \varepsilon^{4}\right)$, where $\varepsilon$ is the wave steepness.

\subsection{Comparison with the arbitrary depth Hasselmann equation}

In order to verify that the kinetic equation derived from the Boussinesq equations is consistent with arbitrary depth Hasselmann equation, a direct comparison of the coupling coefficients is here considered. The coupling coefficient $T_{1,2,3,4}^{a d}$ for arbitrary depth in the Hasselmann equation has the following form (see Janssen and Onorato 2007):

$$
\begin{aligned}
T_{1,2,3,4}^{a d}= & W_{1,2,3,4} \\
& -V_{1,3,1-3}^{(-)} V_{4,2,4-2}^{(-)}\left[\frac{1}{\omega_{3}+\omega_{1-3}-\omega_{1}}+\frac{1}{\omega_{2}+\omega_{4-2}-\omega_{4}}\right] \\
& -V_{2,3,2-3}^{(-)} V_{4,1,4-1}^{(-)}\left[\frac{1}{\omega_{3}+\omega_{2-3}-\omega_{2}}+\frac{1}{\omega_{1}+\omega_{4-1}-\omega_{4}}\right] \\
& -V_{1,4,1-4}^{(-)} V_{3,2,3-2}^{(-)}\left[\frac{1}{\omega_{4}+\omega_{1-4}-\omega_{1}}+\frac{1}{\omega_{2}+\omega_{3-2}-\omega_{3}}\right] \\
& -V_{2,4,2-4}^{(-)} V_{3,1,3-1}^{(-)}\left[\frac{1}{\omega_{4}+\omega_{2-4}-\omega_{2}}+\frac{1}{\omega_{1}+\omega_{3-1}-\omega_{3}}\right] \\
& -V_{1+2,1,2}^{(-)} V_{3+4,3,4}^{(-)}\left[\frac{1}{\omega_{1+2}-\omega_{1}-\omega_{2}}+\frac{1}{\omega_{3+4}-\omega_{3}-\omega_{4}}\right] \\
& -V_{-1-2,1,2}^{(+)} V_{-3-4,3,4}^{(+)}\left[\frac{1}{\omega_{1+2}+\omega_{1}+\omega_{2}}+\frac{1}{\omega_{3+4}+\omega_{3}+\omega_{4}}\right],
\end{aligned}
$$

where the coefficients $V_{1,2,3}^{( \pm)}$are

$$
\begin{aligned}
V_{1,2,3}^{( \pm)}= & \frac{1}{4 \sqrt{2}}\left\{\left[\boldsymbol{k}_{1} \cdot \boldsymbol{k}_{2} \pm q_{1} q_{2}\right]\left(\frac{g \omega_{3}}{\omega_{1} \omega_{2}}\right)^{1 / 2}+\left[\boldsymbol{k}_{1} \cdot \boldsymbol{k}_{3} \pm q_{1} q_{3}\right]\left(\frac{g \omega_{2}}{\omega_{1} \omega_{3}}\right)^{1 / 2}\right. \\
& \left.+\left[\boldsymbol{k}_{2} \cdot \boldsymbol{k}_{3}+q_{2} q_{3}\right]\left(\frac{g \omega_{1}}{\omega_{2} \omega_{3}}\right)^{1 / 2}\right\},
\end{aligned}
$$

with $q_{i}=\omega_{i}^{2} / g . W_{1,2,3,4}$ is given by the following analytical expression:

$$
W_{1,2,3,4}=U_{-1,-2,3,4}+U_{3,4,-1,-2}-U_{3,-2,-1,4}-U_{-1,3,-2,4}-U_{-1,4,3,-2}-U_{4,-2,3,-1}
$$

with

$$
U_{1,2,3,4}=\frac{1}{16}\left(\frac{\omega_{3} \omega_{4}}{\omega_{1} \omega_{2}}\right)^{1 / 2}\left[2\left(k_{1}^{2} q_{2}+k_{2}^{2} q_{1}\right)-q_{1} q_{2}\left(q_{1+3}+q_{2+3}+q_{1+4}+q_{2+4}\right)\right] .
$$


Four-wave resonant interactions in the classical quadratic Boussinesq equations 273

The coupling coefficient in the arbitrary depth Hasselmann equation (see (5.3)) has a similar form as the coupling coefficient in the kinetic equation derived from the Boussinesq equations (see (3.3)). In order to understand the relation between the Hasselmann equation and the Boussinesq kinetic equation we take the shallow water limit of the coupling coefficients, $\left(T^{a d}\right)^{2}$, in the Hasselmann equation. If one takes the leading-order term in the dispersion relation, i.e. $\omega=\sqrt{g h} k$ and substitutes in (5.4) we obtain

$$
\begin{aligned}
V_{1,2,3}^{(+)}=V_{1,2,3}^{(-)}= & \frac{1}{4 \sqrt{2}}\left\{\boldsymbol{k}_{1} \cdot \boldsymbol{k}_{2}\left(\frac{g k_{3}}{c_{0} k_{1} k_{2}}\right)^{1 / 2}+\boldsymbol{k}_{1} \cdot \boldsymbol{k}_{3}\left(\frac{g k_{2}}{c_{0} k_{1} k_{3}}\right)^{1 / 2}\right. \\
& \left.+\boldsymbol{k}_{2} \cdot \boldsymbol{k}_{3}\left(\frac{g k_{1}}{c_{0} k_{2} k_{3}}\right)^{1 / 2}\right\} .
\end{aligned}
$$

One can recognize that $V_{1,2,3}^{(+)}$and $V_{1,2,3}^{(-)}$correspond exactly to the $V_{1,2,3}$ derived from the Boussinesq equations and given in (2.15). It also should be noted that the Hasselmann equation contains an extra term, $W_{1,2,3,4}$, that does not appear in the Boussinesq kinetic equation; nevertheless it is straightforward to show that, using $\omega=\sqrt{g h} k$ in this term, then $W_{1,2,3,4} \sim h k^{4}+O\left(h^{3} k^{6}\right)$; therefore, it is of higher order with respect to the contribution given from $V$ 's terms. This result highlights the fact that both in shallow and deep water the four-wave interaction mechanism can take place and the Hasselmann equation is consistent with the kinetic equation derived from the Boussinesq equations.

\section{Direct energy cascade in the Boussinesq equations}

Here we use a straightforward dimensional analysis of the shallow water kinetic equation in order to investigate on the existence of an inertial range in the wave spectrum in shallow water. The kinetic Boussinesq equations admit as constants of motion both the energy and wave action. If these quantities are globally preserved, it means that across some wavenumber range there must be a flux of energy and wave action. Here we will only concentrate on the direct energy cascade. In order to determine an estimate of the slope of the wave spectrum in the inertial range, we make a dimensional analysis of the kinetic equation

$$
\frac{\partial N_{k}}{\partial t} \sim T^{2} N_{k}^{3} k^{4} \omega^{-1} .
$$

For the direct energy cascade, the flux of energy $\Pi_{k}$ scales as follows:

$$
\Pi_{k} \sim \omega \frac{\partial N_{k}}{\partial t} k^{2} \sim T^{2} N_{k}^{3} k^{6} .
$$

As previously mentioned, to the leading non-trivial order, the coupling coefficient scales as $T \sim k^{2} / h$, and

$$
\Pi_{k} \sim h^{-2} N_{k}^{3} k^{10} ;
$$

therefore, assuming that there is a window of transparency in $k$ space, i.e. a region of constant flux of energy, the flux must be independent of wavenumbers

$$
N_{k} \sim k^{-10 / 3} \quad \text { or } \quad E_{k}=\omega_{k} N_{k} \sim k^{-7 / 3} .
$$


We now move from Cartesian coordinates $\left(k_{x}, k_{y}\right)$ to polar coordinates $(k, \theta)$ so that extra $k$ enters due to the Jacobian of the transformation. The final result is

$$
E_{k} \sim k^{-4 / 3} \sim \omega^{-4 / 3} .
$$

This last result is consistent with the finding of Zakharov (1999). As mentioned in $\S 1$, this power law has been also observed in experimental data by Smith and Vincent (2003) and Kaihatu et al. (2007).

\section{Dicussion and conclusions}

From classical mechanics and dynamical system theory it is well known that an irreversible energy transfer occurs only when exact resonances take place. In flat bottom shallow water equations triad interactions are not exactly resonant; the first non-trivial interaction consists of four interacting waves. Two or more consecutive quasi-resonant triads interactions can be cast as an exact four-wave resonant interaction; therefore when researchers run models based on quasi-resonant triads, they are actually performing higher order nonlinear resonant interactions. As far as we know, there is not an analytical statistical theory based on the three-wave quasi-resonant interaction capable of predicting the slope of the wave spectra. The approach developed here for the Boussinesq equations has allowed us to look at the three-wave non-resonant interactions as four-wave resonant process. In other words, an averaging procedure over the three-wave interactions leads to four-wave resonant interactions.

In this paper, we have discussed a number of issues concerning the propagation of waves in shallow water. We have shown that the four-wave kinetic equation derived from the Boussinesq equations is consistent with the arbitrary depth Hasselmann equation. The time scale of the nonlinear transfer in shallow water can be much faster than the one in deep water. We stress that we choose, as a starting point, the original Boussinesq equations in the present study, not because we believe that these equations represent the best model in shallow water, but because these are the leading-order equations in shallow water which contains quadratic nonlinearity and are dispersive. The physics described by these equations is surely included in higher order models. In the context of the present work it is not a surprise that higher order Boussinesq models can reproduce the modulational instability mechanism. In fact, as one includes higher order terms, the self-interaction coupling coefficient of the four-wave resonant interactions changes sign and the corresponding NLS equation switches from defocusing to focusing.

Formally speaking, there are some limitations in the use of the four-wave kinetic equation in shallow water which are related to the convergence of the expansion in (3.1). This limitations have been discussed in Zakharov (1999). The main problem is related to the fact that for shallow water and steep waves the second-order term in the expansion is not much smaller than the first-order term; therefore, the series cannot be truncated to second or third order. Probably in very shallow water the complete series should be included in order to reconstruct the cnoidal shape of the waves. Higher order terms in the expansion would bring higher order resonant wave-wave interactions. The time scale of nonlinear energy transfer is however dominated by the lowest order approximation, therefore our estimate of the nonlinear time scale should still be reasonable. All these results should be tested directly by comparison with deterministic simulations of the Boussinesq equations or by experiments in wave tank 
Four-wave resonant interactions in the classical quadratic Boussinesq equations 275

facilities. We have also shown in the Appendix that the mechanism of generation of long waves obeys a three-wave kinetic equation from which a nonlinear time scale can be estimated. Such an equation could be used in wave forecasting models to predict the growth of the long-wave components. Clearly, for most applications, the flat bottom hypothesis is just a very poor approximation; we plan therefore to perform a similar analysis including also a non-constant bottom in the Boussinesq equation.

We thank V. Zakharov for valuable discussions and inspiring conversations.

\section{Appendix}

Long-wave generation: asymptotic three-wave resonant interactions in the Boussinesq equations

In the case of non-dispersive waves, i.e. $\omega(k)=\sqrt{g h} k$, three-wave interactions are resonant. Nevertheless, a statistical description of acoustic (non-dispersive) waves is not an easy task because there is not a net separation between the linear and the nonlinear time scales. Avoiding the dispersiveless case, there are basically two type of interactions that can take place, given by the following triads:

$$
\begin{aligned}
& \boldsymbol{k}_{0}=\boldsymbol{k}_{1}+\boldsymbol{k}_{2} \\
& \boldsymbol{k}_{0}=\boldsymbol{k}_{2}-\boldsymbol{k}_{1} .
\end{aligned}
$$

The first one corresponds to the generation of higher harmonics, while the second one, in the limit of $\boldsymbol{k}_{2} \sim \boldsymbol{k}_{1}$, is responsible for the generation of long-wave components. If we are looking for an irreversible transfer of energy, the angular frequencies should satisfy respectively the following relations:

$$
\begin{aligned}
& \omega\left(k_{0}\right)=\omega\left(k_{1}\right)+\omega\left(k_{2}\right) \\
& \omega\left(k_{0}\right)=\omega\left(k_{2}\right)-\omega\left(k_{1}\right)
\end{aligned}
$$

It can be shown that none of the above relations is exactly satisfied if dispersive terms are included. However, there is a difference between the two cases: while in the generation of harmonics the frequency mismatch is of the order of $\beta$ (the small parameter in the Boussinesq approximation), and it is not negligible in dispersive systems, the generation of the long-wave mechanism results in a frequency mismatch which is of higher order with respect to the Boussinesq approximation, therefore can lead to an asympotic resonant interaction. To be more precise, consider two waves $\boldsymbol{k}_{1}$ and $\boldsymbol{k}_{2}$ chosen to be very close to each other and almost parallel, say along the $x$ direction, a very long wavenumber $k_{0}=\Delta k=k_{x 2}-k_{x 1}$ is produced. We now calculate the corresponding frequency mismatch, i.e. $\omega_{0}+\omega_{1}-\omega_{2}=-1 / 2 \sqrt{g h} h^{2} k_{x 2} k_{x 1} \Delta k \simeq$ $-1 / 2 \sqrt{g h}\left(h k_{x 1}\right)^{2} \Delta k$. In shallow water $\left(h k_{x 1}\right)^{2}$ is a small number which is multiplied by $\Delta k$ which is also small if $k_{x 2} \simeq k_{x 1}$. So if $\Delta k / k_{x 1} \sim(k h)^{2} \sim a / h$, the frequency mismatch is of higher order with respect to the Boussinesq approximation and can be neglected within the approximation. The result is that, from a practical point of view, the generation of long waves in shallow water is an irreversible process.

\section{Statistical description of long waves generation}

If we admit that there exists an exact three-wave resonant interaction, then it is possible to build a three-wave kinetic equation. This can be very helpful because it allows us to estimate the time scale of generation of the long waves. Following the standard procedure of wave turbulence theory (Zakharov, L'vov \& Falkovich 1992), 
one can look for an evolution equation for the wave action density and using the random phase approximation and the hypothesis of homogeneity of the wave field, the following evolution equation can be obtained (see for example Zakharov et al. 1992):

$$
\frac{\partial N_{0}}{\partial t}=2 \pi \int\left|V_{0,1,2}\right|^{2}\left(N_{1} N_{2}-N_{0} N_{1}-N_{0} N_{2}\right) \delta\left(\boldsymbol{k}_{1}-\boldsymbol{k}_{0}-\boldsymbol{k}_{2}\right) \delta\left(\omega_{1}-\omega_{0}-\omega_{2}\right) \mathrm{d} \boldsymbol{k}_{12}
$$

where clearly only the resonant contribution has been considered. A dimensional analysis of the above equation shows that the nonlinear time scale of generation of long-wave components is

$$
\tau_{3 N L} \sim \frac{1}{\omega}\left(\frac{1}{(a / h)^{2}(\Delta k / k)}\right) \sim \frac{1}{\omega}\left(\frac{L / \lambda}{(a / h)^{2}}\right),
$$

where $L$ is the length of the long wave and $\lambda$ is the wavelength corresponding to the dominant frequency.

\section{REFERENCES}

Cavaleri, L., Alves, J.-H. G. M., Ardhuin, F., Babanin, A., Banner, M., Belibassakis, K., Benoit, M., Donelan, M., Groeneweg, J., Herbers, T. H. C., Hwang, P., Janssen, P. A. E. M., Janssen, T., Lavrenov, I. V., Magne, R., Monbaliu, J., Onorato, M., Polnikov, V., Resio, D., Rogers, W. E., Sheremet, A., McKee Smith, J., Tolman, H. L., van Vledder, Wolf, J. \& Young, I. 2007 Wave modelling-The state of the art, Prog. Oceanogr. 75(4), 603-674.

Eldeberky, Y. 1996 Nonlinear Transformation of Wave Spectra in the Nearshore Zone. Thesis Delft University of Technology. Also published in the series 'Communications on Hydraulic and Geotechnical Engineering’ (ISSN 0169-6548), Report No. 96-4.

GORMAN, R. M. 2003 The treatment of discontinuities in computing the nonlinear energy transfer for finite-depth gravity wave spectra. J. Atmos. Oceanic Technol. 20, 206-216.

Hasimoto, H. And Ono, H. 1972 Nonlinear modulation of gravity waves. J. Phys. Soc. Jpn. 33, 805-811.

Herbers, T. H. C. \& Burton, M. C. 1997 Nonlinear shoaling of directionally spread waves on a beach. J. Geophys. Res. 102 (C9), 21101-21114.

Herterich, K. \& Hasselmann, K. 1980 A similarity relation for the nonlinear energy transfer in a finite-depth gravity-wave spectrum. J. Fluid Mech. 97, 215-224.

Janssen, T. T., Herbers, T. H. C. \& BAttJes, J. A. 2006 Generalized evolution equations for nonlinear surface gravity waves over two-dimensional topography. J. Fluid Mech. 552, 393-418.

Janssen, P. A. E. M. \& Onorato, M. 2007 The intermediate water depth limit of the Zakharov equation and consequences for wave. J. Phys. Ocean. 10, 2389-2400.

Kaihatu, J. M., Veeramony, J., Edwards, K. L. \& Kirby, J. T. 2007 Asymptotic behaviour of frequency and wave number spectra of nearshore shoaling and breaking waves. J. Geophys. Res. 112, C06016.

KrasitskiI, V. P. 1994 On reduced equations in the Hamiltonian theory of weakly nonlinear surface waves. J. Fluid Mech. 272, 1-20.

Lin, R. Q. \& Perrie, W. 1997 A new coastal wave model. Part III. Nonlinear wave-wave interaction. J. Phys. Ocean. 27, 1813-1826.

Madsen, P. A., Bingham, H. B. \& LiU, H. 2002 A new Boussinesq method for fully nonlinear waves from shallow to deep water. J. Fluid Mech. 462, 1-20.

Madsen, P. A. \& Schaffer, H. A. 1998 Higher-order Boussinesq-type equations for surface gravity waves: derivation and analysis. Phil. Trans. R. Soc. Lond. A 356, 3123-3184.

MeI, C. C. 2000 The Applied Dynamics of Ocean Surface Waves. John Wiley and Sons.

Smith, J. M. \& Vincent, C. L. 2003 Equilibrium ranges in surf zone wave spectra. J. Geophys. Res.-Ocean 108, 3366, published 26 November 2003, doi:10.1029/2003JC001930.

Stiassnie, M. \& Shemer, L. 1984 On modifications of the Zakharov equation for surface gravity waves. J. Fluid Mech. (1984) 143, 47-67. 
Four-wave resonant interactions in the classical quadratic Boussinesq equations 277

Whitham, G. B. 1974 Linear and Nonlinear Waves, John Wiley and Sons, 636 pp.

ZAKHAROV, V. 1968 Stability of periodic waves of finite amplitude on the surface of a deep fluid. $J$. Appl. Mech. Tech. Phys. 9, 190-194.

ZAKHAROV, V. E. 1998 Weakly nonlinear waves on the surface of an ideal finite depth fluid. In Nonlinear Waves and Weak Turbulence (ed. V. E. Zakharov), American Mathematical Society.

ZAKHAROV, V. E. 1999 Statistical theory of gravity and capillary waves on the surface of a finite-depth fluid. Eur. J. Mech. B/Fluids 18, 327-344.

Zakharov, V. E., L'vov, V. S. \& Falkovich G. 1992 Kolmogorov Spectra of Turbulence. Springer.

ZaKharov, V. E. \& Schulman, E. I. 1988 On additional motion invariants of classical Hamiltonian wave systems. Phys. D 29, 283-320. 